Research Article

\title{
Microstructure and Damping Property of Polyurethane Composites Hybridized with Ultraviolet Absorbents
}

\author{
Jiang Chang, ${ }^{1,2}$ Bing Tian $\mathbb{D}^{1},{ }^{1} \mathrm{Li} \mathrm{Li}^{1}{ }^{1}$ and Yufeng Zheng ${ }^{1}$ \\ ${ }^{1}$ Institute of Materials Processing and Intelligent Manufacturing, College of Materials Science and Chemical Engineering, Harbin \\ Engineering University, Harbin 150001, China \\ ${ }^{2}$ College of Light Industry and Textile Engineering, Qiqihar University, Qiqihar 161006, China
}

Correspondence should be addressed to Bing Tian; tianbing@hrbeu.edu.cn

Received 7 September 2017; Revised 30 November 2017; Accepted 6 December 2017; Published 10 January 2018

Academic Editor: Marino Lavorgna

Copyright (c) 2018 Jiang Chang et al. This is an open access article distributed under the Creative Commons Attribution License, which permits unrestricted use, distribution, and reproduction in any medium, provided the original work is properly cited.

\begin{abstract}
This article investigated the microstructure and damping property of TPU composites with different contents of ultraviolet absorbents. It can be found that the ultraviolet absorbents formed fiber-shaped precipitations in the TPU matrix. The UV-328 was randomly distributed in the matrix and exhibited a weak interfacial bonding with the matrix. In comparison, the UV-329 was well embedded in the matrix and formed a relatively better interfacial bonding and compatibility with the TPU matrix. The damping factor $\tan \delta$ of both 328-composites and 329-composites had been reduced gradually with increasing content of ultraviolet absorbents at the glass transition temperature range due to the fact that the ultraviolet absorbents were in the crystalline state which decreased the volume content of the viscoelastic TPU matrix. But the tan $\delta$ increased at the temperature range of higher than the glass transition temperature, which should be related to the dominance of interfacial frictions between the ultraviolet absorbents and the matrix on the energy absorption.
\end{abstract}

\section{Introduction}

Polyurethane has been widely investigated in the past few years due to its comprehensive properties of high hydrolysis resistance, wear resistance, good flexibility, and damping ability [1-4]. The high damping property of polymers is mainly observed during glass transition process, in which the mechanical energy caused by vibrational motion of molecular chains is transferred to heat energy. Beyond this range, the polymer often presents a low damping property. Therefore, the application for polymers is often restricted to a narrow temperature range associated with glass transition. Generally, low elastic modulus of the polymers also restricts their engineering applications to some extent. Thus, properly extending the damping range and enhancing the modulus of polymers are meaningful for the practical applications. Several methods, such as mechanical blending [5], interpenetrating polymer networks (IPNs) $[6,7]$ and forming organic hybrid composites composed of polar polymers and functional organic small molecules [8], have been proposed and investigated to improve the damping ability of the polymers. In comparison, the last method of forming intermolecular hydrogen bonds to improve the damping properties has presented a higher cost-effective prospect. The DZ (N, $\mathrm{N}^{\prime}$-dicyclohexyl-benzothiazole-2-sulfonamide), AO-80 (3,9-bis $\{1,1$-dimethyl-2[ $\beta$ (3-tert-butyl-4-hydroxy-5methylphenyl)propionyloxy] ethyl $\}-2,4,8,10$-tetraoxaspiro [5,5]-undecane), AO-60 (tetrakis[methylene-3-(3-5-ditert-butyl-4-hydroxy phenyl) propionyloxy] methane), etc. hindered phenol have been selected as the functional organic small molecules to be added in the chlorinated polyethylene matrix [8-10], and an apparent improvement of the damping factor of the polymer matrix has been observed. However, little information on the effect of organic hybridizing on the damping property of polyurethane is available. In this article, the ultraviolet absorbent with functional organic small molecules has been added into the thermoplastic polyurethanes (TPUs) to investigate the effect of organic hybridizing on the microstructure and damping property of the TPU. 


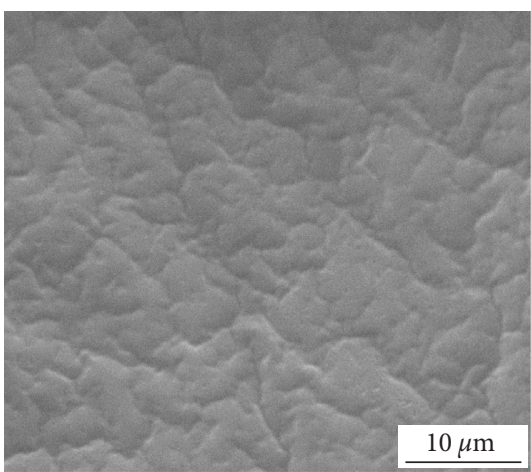

(a)

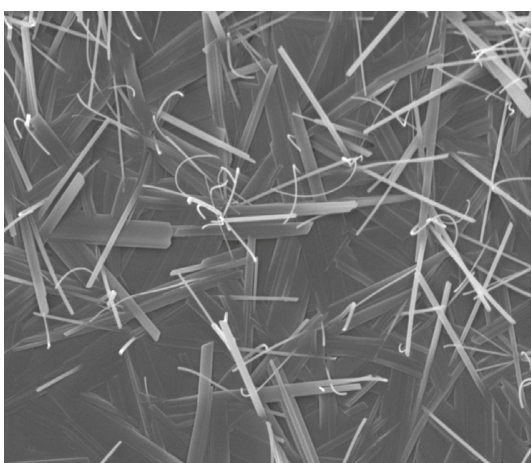

(c)

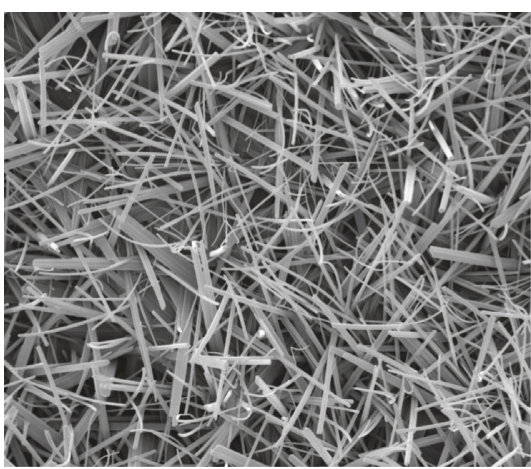

(e)

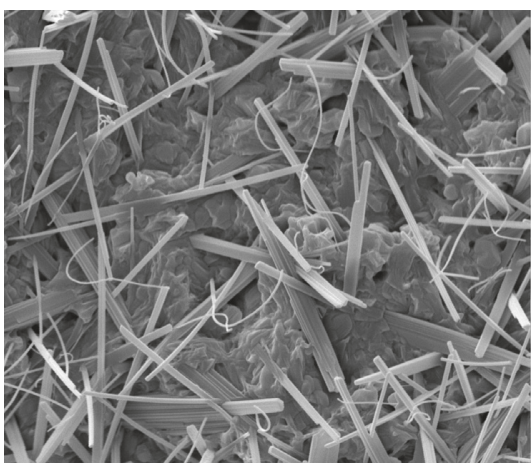

(g)

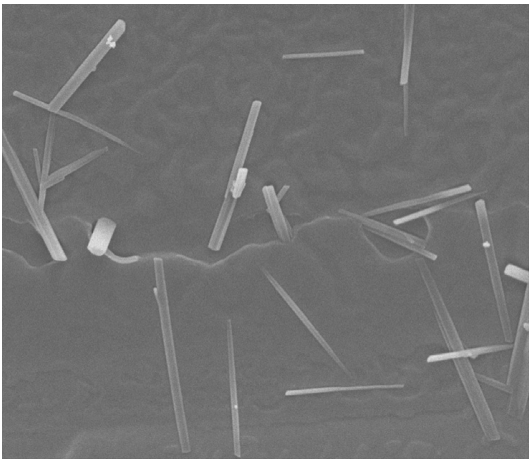

(b)

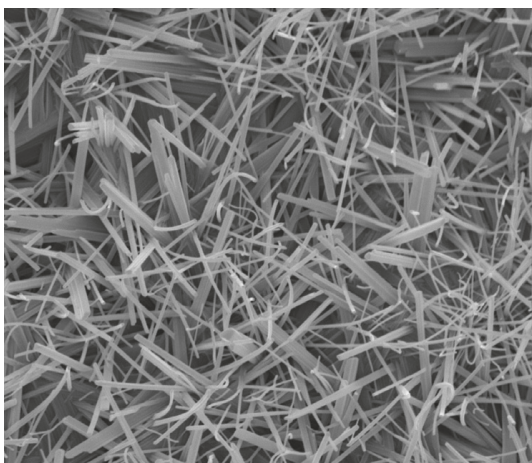

(d)

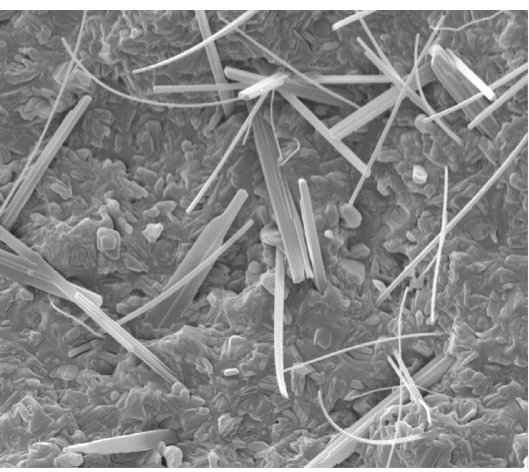

(f)

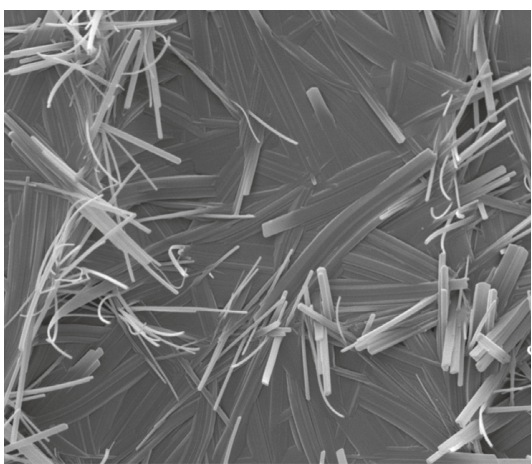

(h)

FIGURE 1: SEM images of fracture surfaces of (a) TPU and 328-composites with different mass ratios of 328: (b) 10\%, (c) 20\%, (d) 25\%, (e) $30 \%$, (f) $40 \%$, (g) $50 \%$, and (h) $60 \%$. 


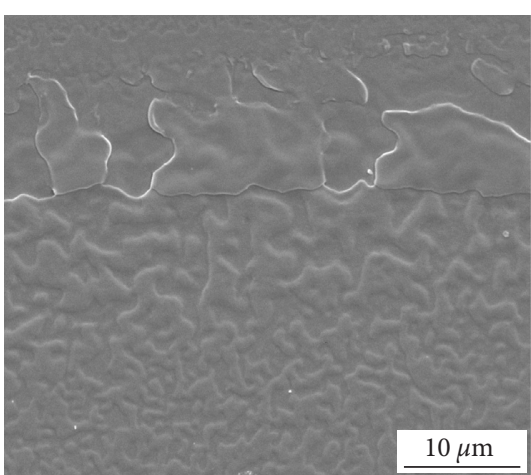

(a)

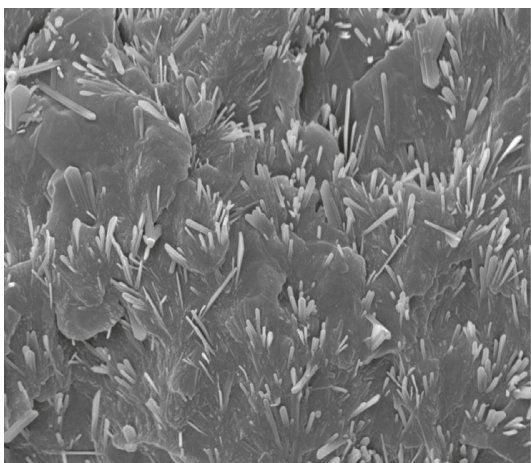

(c)

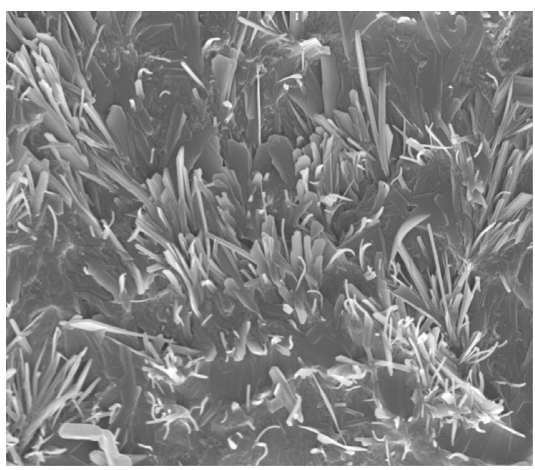

(e)

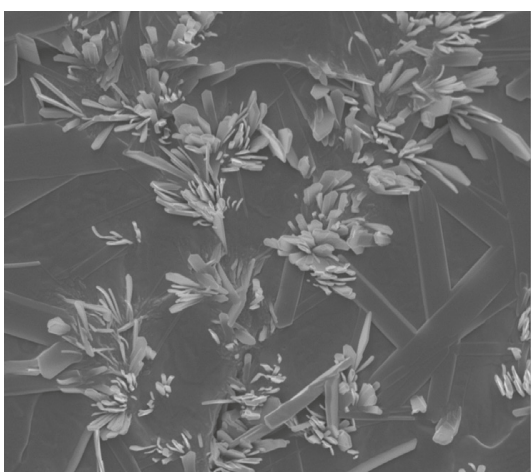

(b)

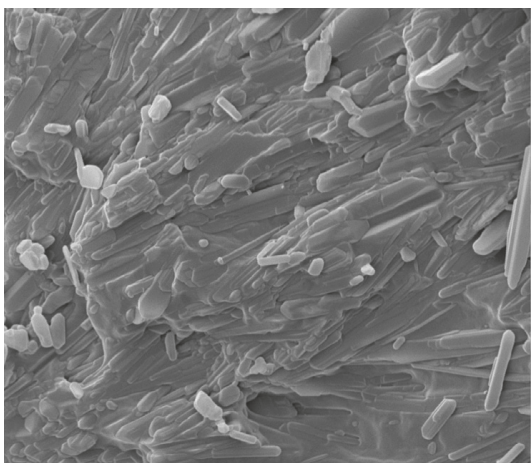

(d)

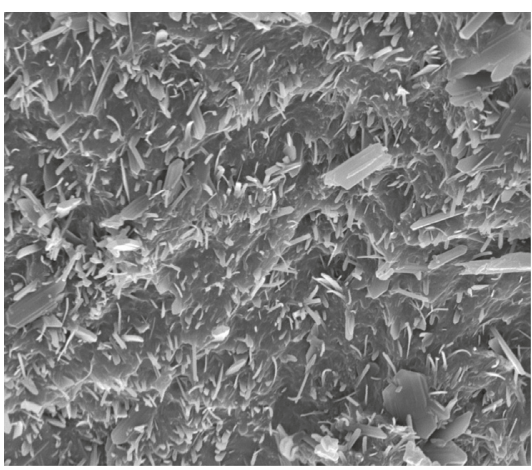

(f)

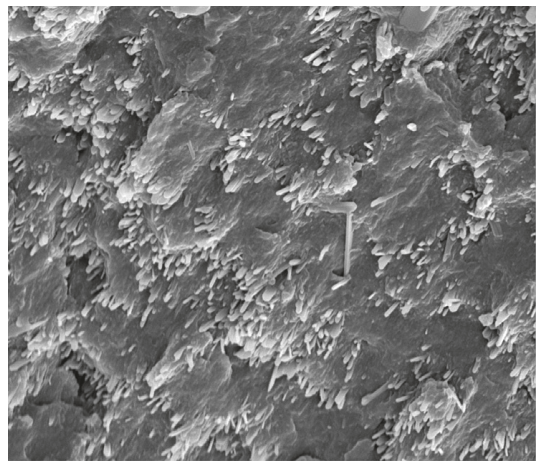

(g)

FigURE 2: SEM images of fracture surfaces of 329-composites with different mass ratios of 329: (a) 10\%, (b) 20\%, (c) 25\%, (d) $30 \%$, (e) 40\%, (f) $50 \%$, and (g) $60 \%$. 


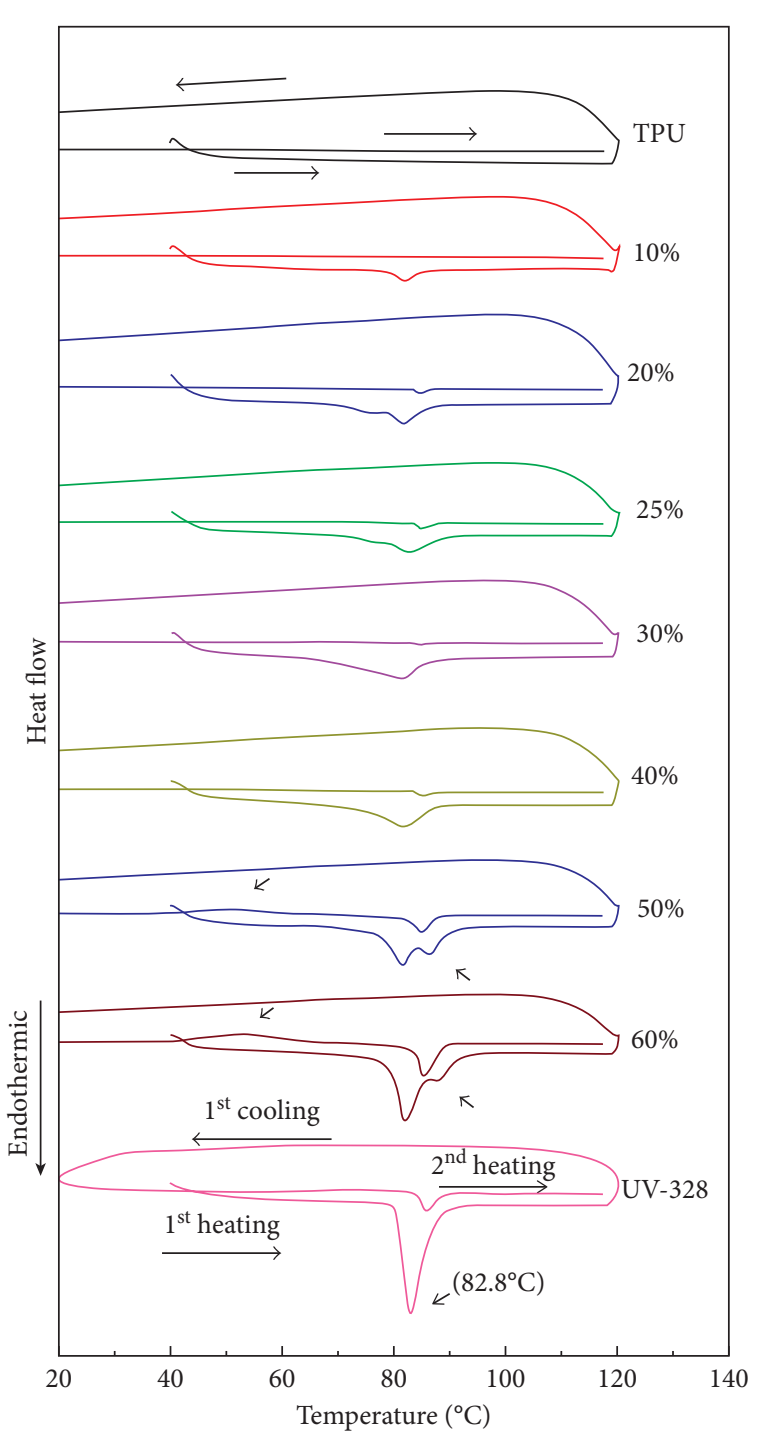

(a)

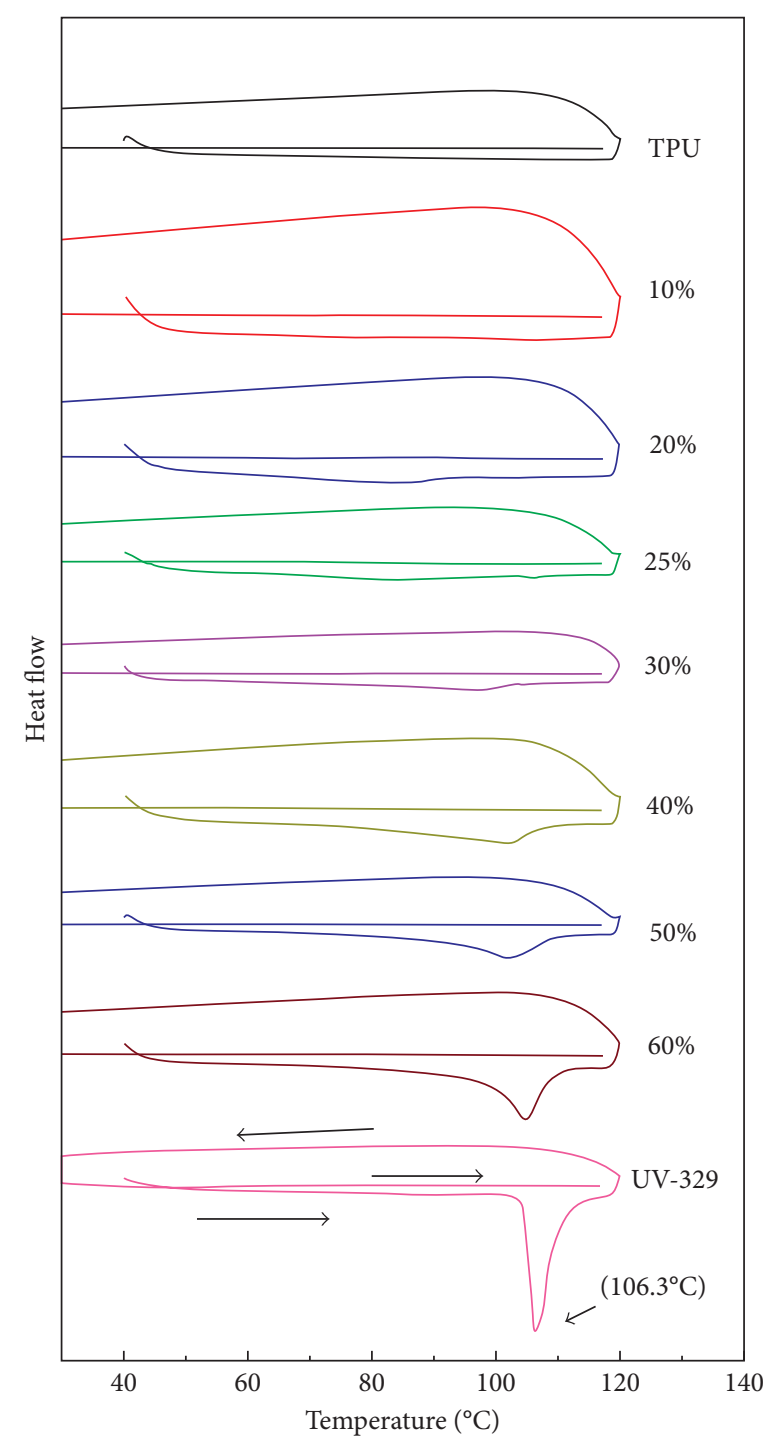

(b)

Figure 3: DSC curves of (a) 328-composites and (b) 329-composites with different mass ratios of UV-absorbents.

\section{Experimental Details}

The ultraviolet (UV) absorbents 2-( $2^{\prime}$-hydroxy- $3^{\prime}, 5^{\prime}$-di-tertamylphenyl) benzotriazole UV-328 $\left(\mathrm{C}_{22} \mathrm{H}_{29} \mathrm{~N}_{3} \mathrm{O}\right)$ and 2- $\left(2^{\prime}-\right.$ hydroxy-5'-tert-octylphenyl) benzotriazole UV-329 $\left(\mathrm{C}_{20} \mathrm{H}_{25} \mathrm{~N}_{3} \mathrm{O}\right)$ (powders supplied by Nippon Kasei Chemical Co., Ltd., Japan) were selected as the functional organic small molecules to be added into the WHT-8185 polyether thermoplastic polyurethane (TPU, supplied by Yantai wanhua polyurethanes Co., Ltd., China), respectively, with a mass ratio of $10-60 \%$. The melting point of UV-328 and UV-329 is at $80-83^{\circ} \mathrm{C}$ and $101-106^{\circ} \mathrm{C}$, respectively. Both UV328 and UV-329 belong to benzotriazole-type chemicals, and the difference between them is the different substituent adjacent to the hydroxyl group. The typical parameters of WHT-8185 obtained from the manufacturer are as follows: glass transition temperature: $\sim-45^{\circ} \mathrm{C}$, tensile strength: $26 \mathrm{MPa}$, elongation: 500\%, hardness: 85 (shore A), and processing temperature: $180-195^{\circ} \mathrm{C}$. Firstly, the TPU particles were dissolved in the solvent mixture with a volume ratio of $1: 1$ of $\mathrm{N}, \mathrm{N}$-dimethylformamide (DMF) and tetrahydrofuran (THF) and heated in a vacuum oven at $100^{\circ} \mathrm{C}$ for $40 \mathrm{~min}$ to accelerate dissolution. Then, the UVabsorbents were dissolved into the above mixture and ultrasonically dispersed at $35^{\circ} \mathrm{C}$ for $0.5 \mathrm{~h}$. Finally, the mixture solution was poured into a mold and then dried in a vacuum oven to fully evaporate the dissolvent of DMF and THF to obtain the UV-absorbent/TPU composite thin films. For simplicity, thereafter, the composites containing UV-328 and UV-329 absorbents were denoted as 328-composites and 329-composites, respectively.

The damping curves were recorded by a dynamic mechanical analyzer $\left(\mathrm{DMA}^{+} 450\right)$ under a tension mode, in which the frequency was $10 \mathrm{~Hz}$, the vibration amplitude was $5 \mu \mathrm{m}$, and the heating rate was $3^{\circ} \mathrm{C} \mathrm{min}^{-1}$. The dimension of samples for DMA testing was $\sim 30 \times 8 \times 0.3 \mathrm{~mm}^{3}$. DSC 


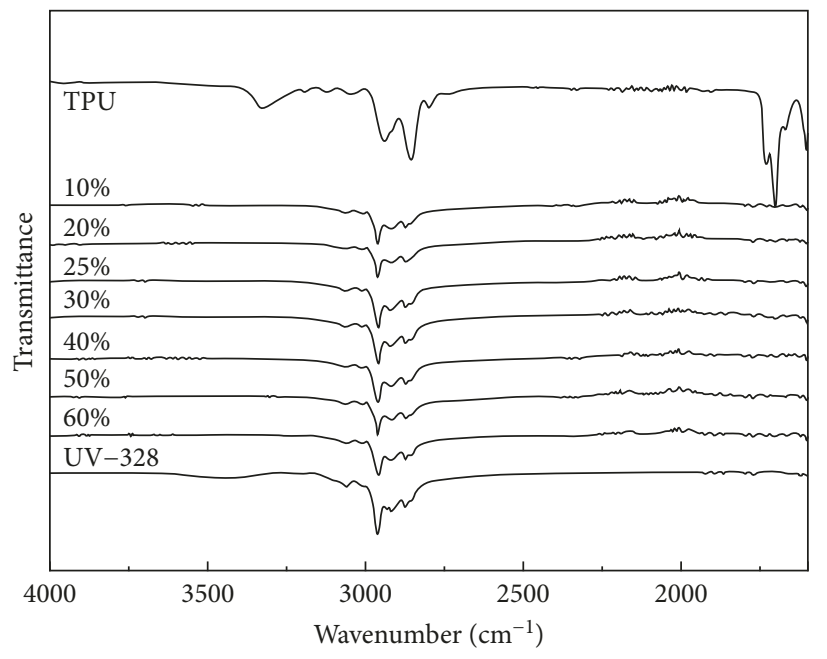

(a)

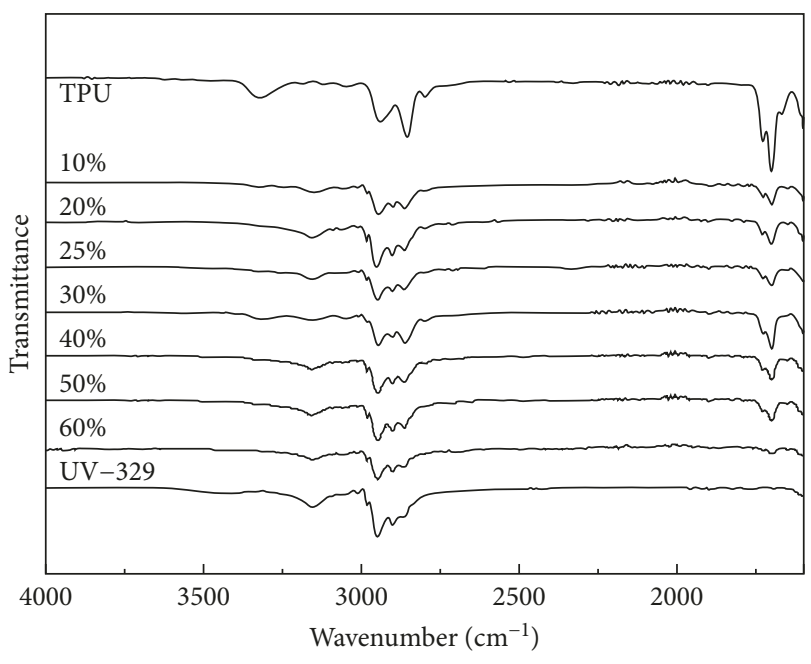

(b)

FIGURE 4: FTIR spectra of (a) 328-composites and (b) 329-composites with different mass ratios of UV-absorbents.

measurements were performed in a TA-Q200 apparatus with a heating/cooling rate of $20^{\circ} \mathrm{C} \mathrm{min}^{-1}$. FTIR test was performed in a Perkin-Elmer Spectrum-100 fourier transform infrared spectrometer. IR spectrum was obtained by scanning 32 times at the resolution of $4 \mathrm{~cm}^{-1}$ within the wavenumber range of $450-4000 \mathrm{~cm}^{-1}$. IR spectrum of the $\mathrm{UV}$-absorbent powders was obtained using the $\mathrm{KBr}$ pallet method, and IR spectrum of UV-absorbent/TPU composite films was obtained using the attenuated total reflection (ATR) method. Microstructure of the samples was observed using FEI Quanta200 scanning electron microscope (SEM).

\section{Results and Discussion}

The TPU composites exhibit a very large plasticity at room temperature and are difficult to be fractured, so the composites are bent to fracture under liquid nitrogen to observe microstructure of the samples. Figure 1 shows fracture surface of the composites with different mass ratios of 328 UV-absorbents. All the micrographs have the same magnification to that of Figure 1(a). It can be seen that the pure TPU presents a flat fracture surface with a single phase, as shown in Figure 1(a). After adding 328 for 10\% (Figure 1(b)), the composite is found to be composed of two phases, including a flat matrix and some short fibers that should be 328 absorbents precipitated from the solution mixture after removing the solvents. With increasing 328 from $20 \%$ to $30 \%$ (Figures $1(\mathrm{c})-1(\mathrm{e})$ ), it is seen that the fiber volume has been greatly increased and the matrix was nearly fully covered and cannot be seen on the surface. However, continue increasing 328 to $40 \%-60 \%$ (Figures $1(\mathrm{f})-1(\mathrm{~h})$ ), it seems that the 328 fiber volume is decreased and the TPU matrix can be found, which could be caused by the fact that the distribution of 328 fibers is not homogeneous in the composites and these composites mainly fractured on the areas with few 328 fibers.

For the 329-composites, as shown in Figure 2, it is different from that of the 328-composites. For the $10 \%$ composite (Figure 2(a)), there is no 329 phases observed on the surface. For the composites with $20 \% 329$, the petaloid 329 phases can be found, as shown in Figure 2(b). Continue increasing 329 from $25 \%$ to $60 \%$ (Figures $2(\mathrm{c})-2(\mathrm{~g})$ ), the fracture surface becomes rougher gradually as compared to the pure TPU, but there is no apparent two-phase separation like 328-composites observed on the surface and the 329 phases seem to be mostly embedded in the TPU matrix. The above microstructure results demonstrate that the UV-329 should have a better compatibility with the TPU matrix than the UV-328.

Figures 3(a) and 3(b) show the DSC results of the 328composites and 329-composites, respectively. All the samples are subjected to a thermal cycle of heating, cooling, and reheating. As shown in Figure 3(a), it can be seen that there is no any thermal phenomenon observed for the pure TPU at this thermal cycle. For the pure 328, an endothermic peak centered at around $82.8^{\circ} \mathrm{C}$ during first heating process can be found, which should stand for the melting of the UV-328. Upon cooling, there is no thermal event observed, but one much weaker endothermic peak than that of the first heating at similar temperature is observed during the second heating. The occurrence of the weak endothermic peak means the melting of some crystalline 328 phases that should recrystallize during the cooling process although the thermal event associated with it is not detected on cooling. For the composites with 328 of $>10 \%$, these two endothermic peaks associated with the melting of 328 phases are also observed, and these two peaks become stronger gradually with increasing 328 content. In addition, it is noted that, for the $50 \%$ and $60 \%$ composites, except for the abovementioned two endothermic peaks, an extra endothermic peak during first heating and an exothermic peak on the second heating are observed, as indicated by arrows. From Figure 1, it can be seen that the 328 fibers mainly have two forms in the composites, one is "free form" that has almost no interaction with the TPU matrix (Figures 1(d) and 1(e)), and the other is "embedding form" that has some interfacial bonding with the matrix 


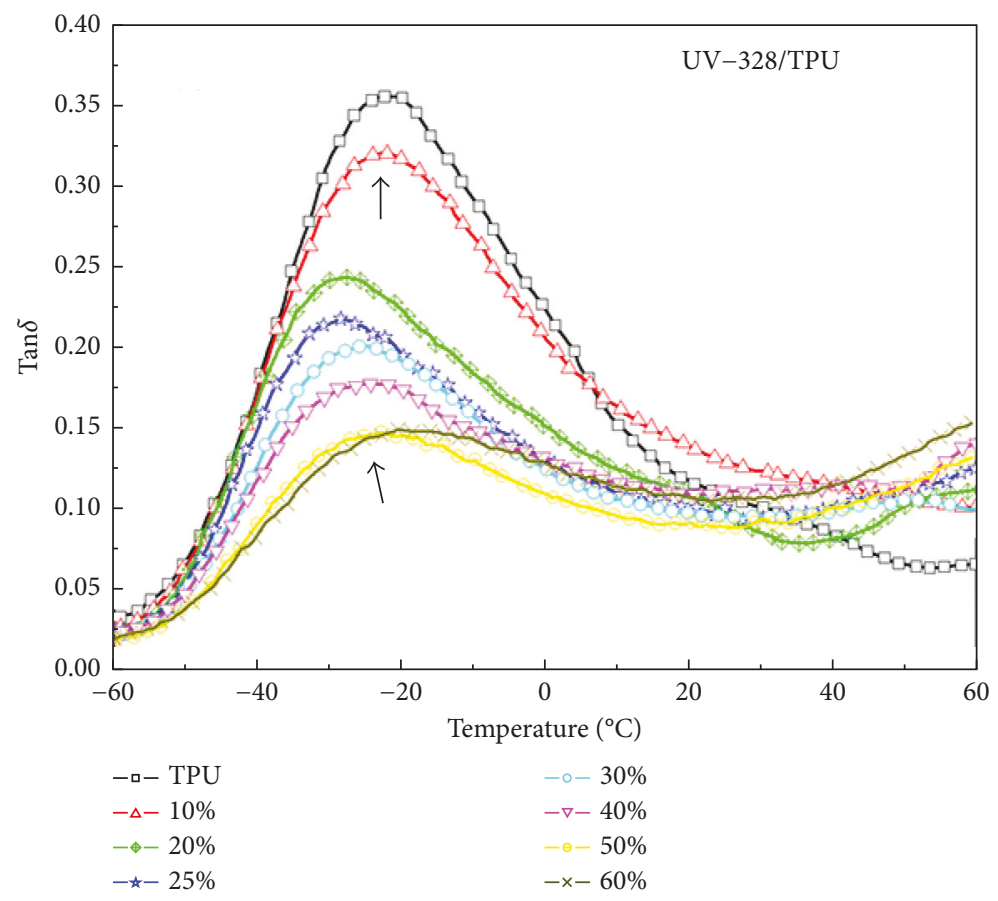

(a)

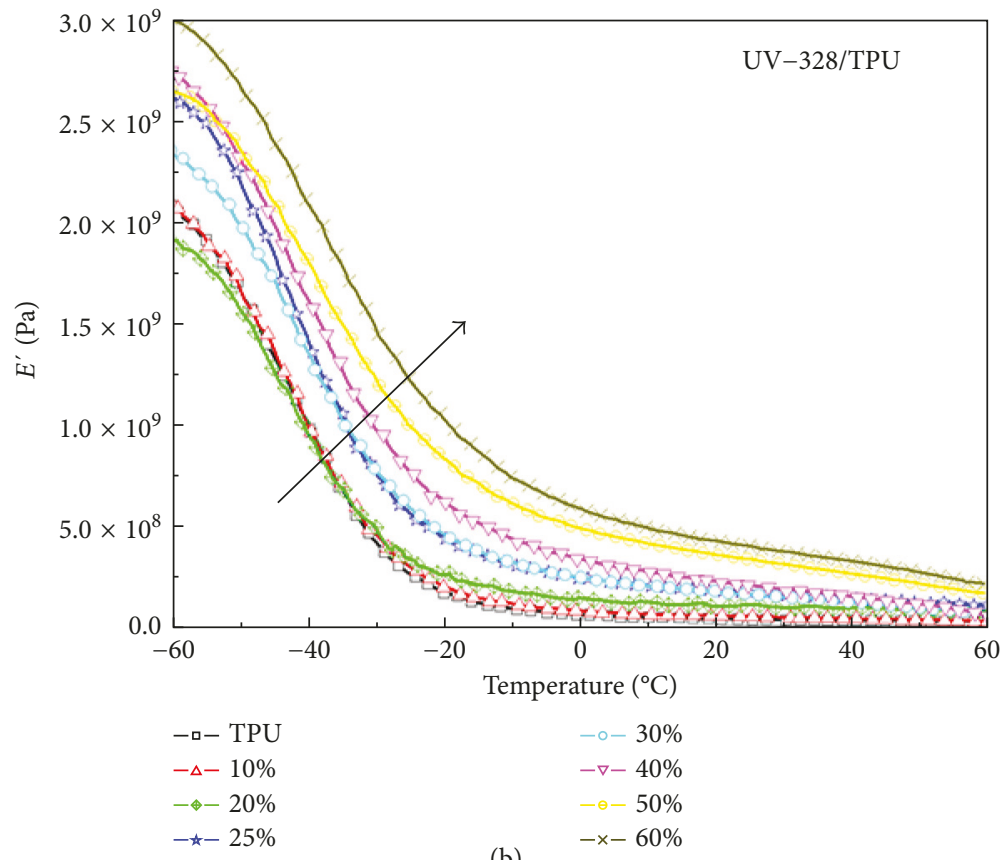

Figure 5: Temperature dependence of (a) $\tan \delta$ and (b) $E^{\prime}$ for the 328-composites.

(Figures 1(c) and 1(h)). Therefore, during the first heating, the second endothermic peak indicated by the arrow should be related to the melting of the UV fiber with "embedding form" and the exothermic peak indicated by the arrow on the second heating means the debonding process between the "embedding form" UV fiber and the TPU matrix.

For the 329-composites, it is a little different from that of the 328-composites, as shown in Figure 3(b). Only one endothermic peak centered at $106.3^{\circ} \mathrm{C}$ associated with melting of 329 phases is observed during the first heating process and no endothermic peak is found on the second heating, meaning that the 329 phase is also in the crystalline state and no recrystallization happened on subsequent cooling. In addition, it is found that, unlike 328-composite, the melting peak on the first heating process for the 329composite with 329 content of $<30 \%$ cannot be found. The 329-composite exhibits a relatively wide melting peak with low latent heat as compared to the 328-composite. 


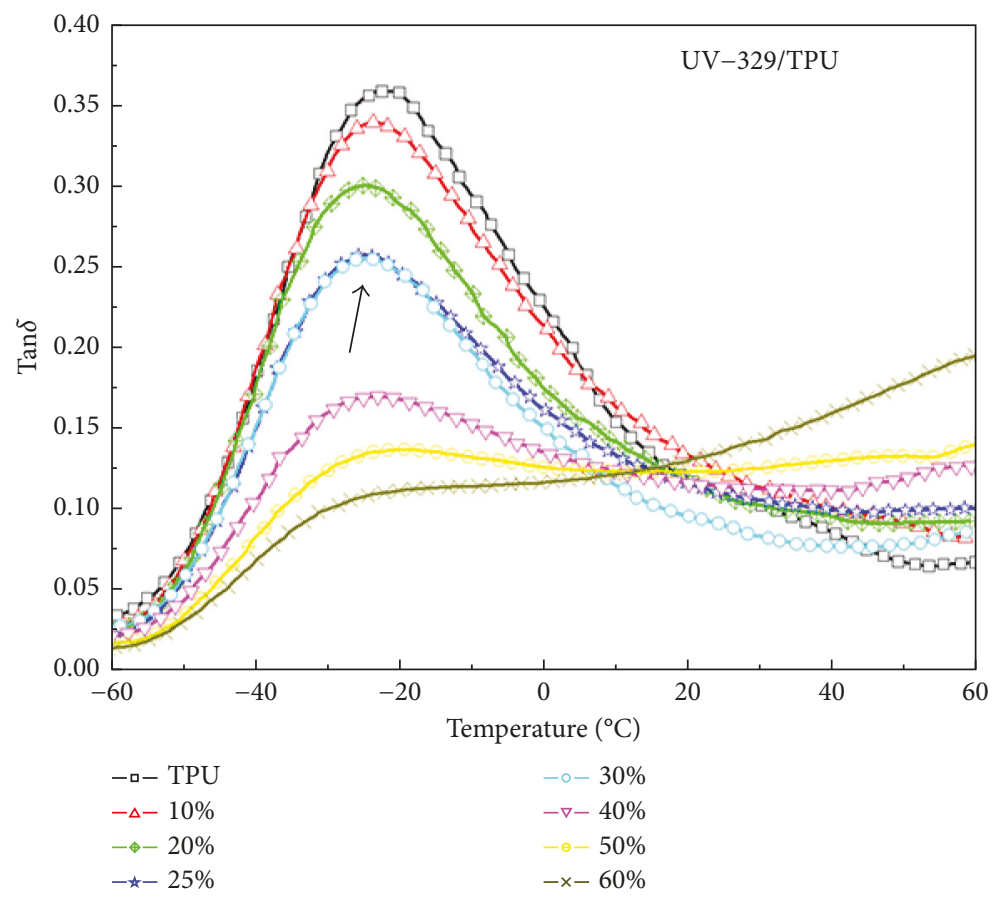

(a)

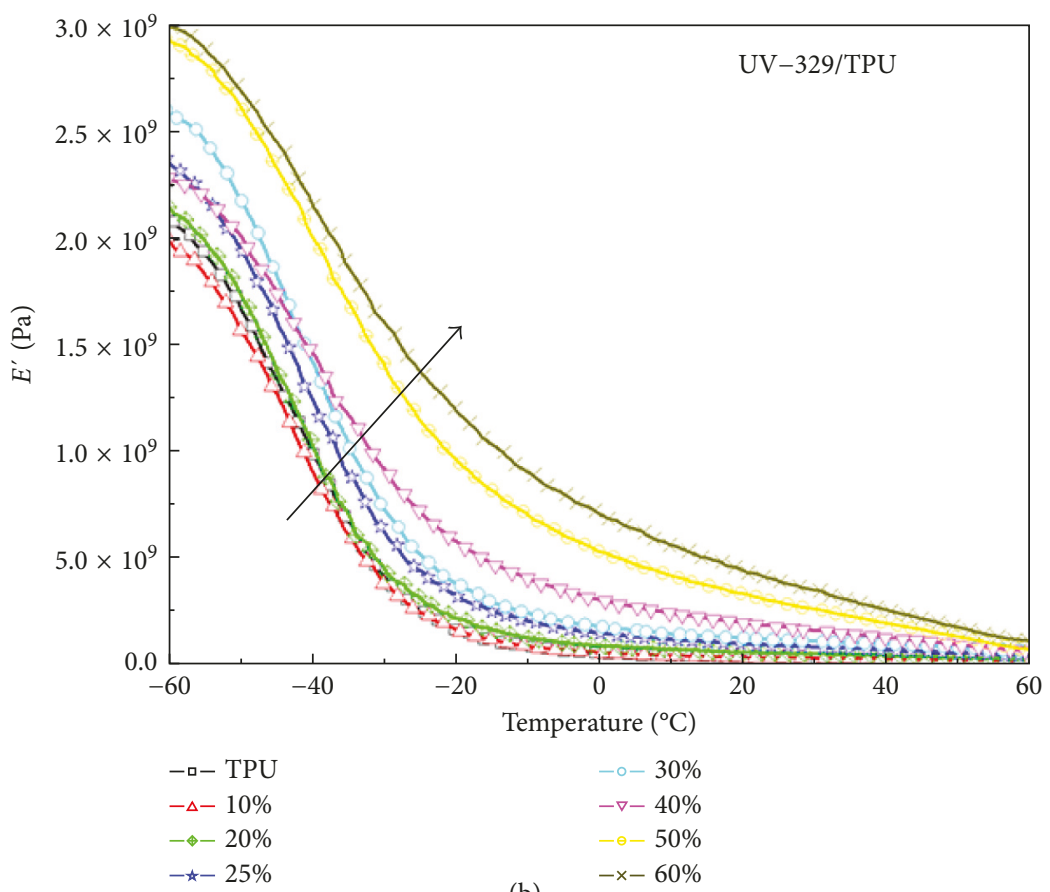

Figure 6: Temperature dependence of (a) $\tan \delta$ and (b) $E^{\prime}$ for the 329-composites.

Therefore, the absence of melting peaks should be caused by the low melting latent heat of the 329-composite with 329 content of $<30 \%$. The above results demonstrate that the $328 / 329$ UV-absorbents in the as-prepared composites are mainly in the crystalline state at room temperature.

Figures 4(a) and 4(b) show the infrared spectra of the 328-composites and 329-composites, respectively. It can be seen that, for 328-composites, they are mainly reflected by the absorption peaks that belong to the UV-328, as shown in Figure 4(a). With increasing the content of 328, there is no apparent change of the spectra. This should be caused by the fact that a large amount of 328 phases formed on the sample surface that covered the TPU matrix (Figure 1) and resulted in the disappearance of the absorption peaks for the matrix. In contrast, for the 329-composites, they are mainly composed of the absorption peaks of 329 and TPU matrix, which 
is also in agreement with the SEM results shown in Figure 2, in which the 329 phases is well embedded in the TPU matrix. However, the intermolecular hydrogen bonding observed in the organic hybrid materials [8-10] was not detected in the present composites. The undetected intermolecular hydrogen bonding for both composites could result from two reasons: one is the crystalline UV absorbents that are not favorable for the formation of hydrogen bonding with TPU matrix, and the other is the less functional group in these UV absorbents.

Figure 5(a) shows the temperature dependence curve of the damping factor $\tan \delta$ for the 328 -composites. It is seen that the composites exhibit a similar $\tan \delta$ curve to that of the pure TPU; that is, the $\tan \delta$ firstly increased with increasing temperature and then decreased and reached the maximum at the glass transition temperature $\left(\sim-20^{\circ} \mathrm{C}\right)$ of the matrix. It is clear that the $\tan \delta$ maximum is gradually reduced with increasing the 328 content for the composites. However, it is also noted that the $\tan \delta$ seems to be gradually increased to some extent with increasing 328 content when the temperature is higher than $30^{\circ} \mathrm{C}$ when the TPU matrix is in a full elastomeric state. Therefore, it is suggested that the damping mechanism is different for these two temperature ranges. The energy absorption for the present composites should come from two ways, the first is the intrinsic damping of the TPU matrix caused by molecular movements, and the other is the interfacial friction between the matrix and 328 phases [11]. During the glass transition process $\left(-60\right.$ to $\left.30^{\circ} \mathrm{C}\right)$, the former way plays a predominant role; therefore, the $\tan \delta$ was gradually decreased with increasing 328 that diluted the TPU matrix. After glass transition, the energy harvesting coming from TPU has been minimum and the interfacial friction between the matrix and 328 phases has become predominant, thus resulting in the increase of $\tan \delta$ when the matrix is in the elastomeric state.

Figure 5(b) shows the storage modulus $\left(E^{\prime}\right)$ curves with variation of temperature for the 328-composites. It can be seen that the $E^{\prime}$ exhibits a sudden drop during glass transition from the glassy state to the elastomeric state. The $E^{\prime}$ of composites has similar changing trend to that of the TPU. It is noted that the $E^{\prime}$ has been increasingly enhanced with increasing 328 content, which should be caused by the reinforcing effect of 328 phases on the TPU matrix. The 328 phases are in the crystalline state and harder than the TPU matrix, therefore resulting in the reinforcement of the matrix.

Figures 6(a) and 6(b) show the $\tan \delta$ curves and $E^{\prime}$ curves with variation of temperature for the 329-composites, respectively. It can be seen that they are similar to the results obtained in Figure 5 for the 328-composites. The $\tan \delta$ maximum of the composites is gradually reduced with increasing 329 content during glass transition range, whereas the $\tan \delta$ is enhanced when the matrix is at a full elastomeric state $\left(>20^{\circ} \mathrm{C}\right)$. It is noted that, for the 329 -composites with $60 \% 329$, the $\tan \delta$ at temperature of $>20^{\circ} \mathrm{C}$ is even higher than that at the glass transition temperature range. For the $E^{\prime}$, similar to the 328-composites, the $E^{\prime}$ of the 329-composites is gradually enhanced due to the reinforcing effect of the crystalline 329 phases on the TPU matrix. Based on the above results, it is concluded that the addition of 328 and 329 phases reduced the damping capacity at the glass transition range of the composites due to the dilution of TPU matrix but increased the damping property when the TPU matrix is at elastomeric state because of the interfacial frictions between the matrix and UV-absorbents. Meanwhile, the addition of UV-absorbents enhanced the TPU matrix and increased the elastic modulus of the composites.

\section{Conclusions}

The microstructure and damping property of polyurethane composites with different contents of UV-absorbents (UV328 and UV-329) are investigated in this article. It is found that the UV-328 absorbents formed fiber-shaped phases in the TPU matrix and exhibited a very weak compatibility with the matrix, whereas the UV-329 absorbents are embedded in the matrix and form a relatively better compatibility with the matrix. Both 328 and $329 \mathrm{UV}$-absorbents are in the crystalline state in the composites at room temperature. The $\tan \delta$ for the composites at glass transition range is gradually reduced with increasing content of UV-absorbents due to the dilution of matrix by the UV-absorbents. But the $\tan \delta$ for the composites is increasingly enhanced with increasing $\mathrm{UV}$-absorbents when the matrix is at a full elastomeric state, which is attributed to the interfacial frictions between the $\mathrm{UV}$-absorbents and the matrix. The storage modulus of the composites is gradually enhanced with increasing content of $\mathrm{UV}$-absorbents due to the reinforcing effect of the crystalline UV-absorbents on the TPU matrix.

\section{Conflicts of Interest}

The authors declare that they have no conflicts of interest.

\section{References}

[1] A. Eceiza, M. D. Martin, K. de la Caba et al., "Thermoplastic polyurethane elastomers based on polycarbonate diols with different soft segment molecular weight and chemical structure: mechanical and thermal properties," Polymer Engineering and Science, vol. 48, no. 2, pp. 297-306, 2008.

[2] B. C. Chun, T. K. Cho, M. H. Chong, and Y. C. Chung, "Structure-property relationship of shape memory polyurethane cross-linked by a polyethyleneglycol spacer between polyurethane chains," Journal of Materials Science, vol. 42, no. 21, pp. 9045-9056, 2007.

[3] W. L. Li, J. L. Liu, C. W. Hao, K. Jiang, D. F. Xu, and D. J. Wang, "Interaction of thermoplastic polyurethane with polyamide 1212 and its influence on the thermal and mechanical properties of TPU/PA1212 blends," Polymer Engineering and Science, vol. 48, no. 2, pp. 249-256, 2008.

[4] M. Nakamura, Y. Aoki, G. Enna, K. Oguro, and H. Wada, "Polyurethane damping material," Journal of Elastomers and Plastics, vol. 47, no. 6, pp. 515-522, 2015.

[5] N. Yamada, S. Shoji, H. Sasaki et al., "Developments of high performance vibration absorber from poly(vinyl chloride)/chlorinated polyethylene/epoxidized natural rubber blend," Journal of Applied Polymer Science, vol. 71, no. 6, pp. 855-863, 1999. 
[6] X. Yu, G. Gao, J. Wang, F. Li, and X. Tang, "Damping materials based on polyurethane/polyacrylate IPNs: dynamic mechanical spectroscopy, mechanical properties and multiphase morphology," Polymer International, vol. 48, no. 9, pp. 805-810, 1999.

[7] C. L. Qin, W. M. Cai, J. Cai, D. Y. Tang, J. S. Zhang, and M. Qin, "Damping properties and morphology of polyurethane/vinyl ester resin interpenetrating polymer network," Materials Chemistry and Physics, vol. 85, no. 2-3, pp. 402-409, 2004.

[8] C. F. Wu and S. Akiyama, "Enhancement of damping performance of polymers by functional small molecules," Chinese Journal of Polymer Science, vol. 20, pp. 119-127, 2002.

[9] C. F. Wu, Y. Otani, N. Namiki, H. Emi, K. Nitta, and S. Kubota, "Dynamic properties of an organic hybrid of chlorinated polyethylene and hindered phenol compound," Journal of Applied Polymer Science, vol. 82, no. 7, pp. 17881793, 2001.

[10] C. Zhang, P. Wang, C. Ma, and M. Sumita, "Damping properties of chlorinated polyethylene-based hybrids: effect of organic additives," Journal of Applied Polymer Science, vol. 100, no. 4, pp. 3307-3311, 2006

[11] J. Suhr, N. Koratkar, P. Keblinski, and P. Ajayan, "Viscoelasticity in carbon nanotube composites," Nature Materials, vol. 4, no. 2, pp. 134-137, 2005. 


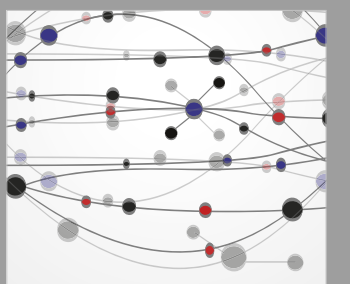

The Scientific World Journal
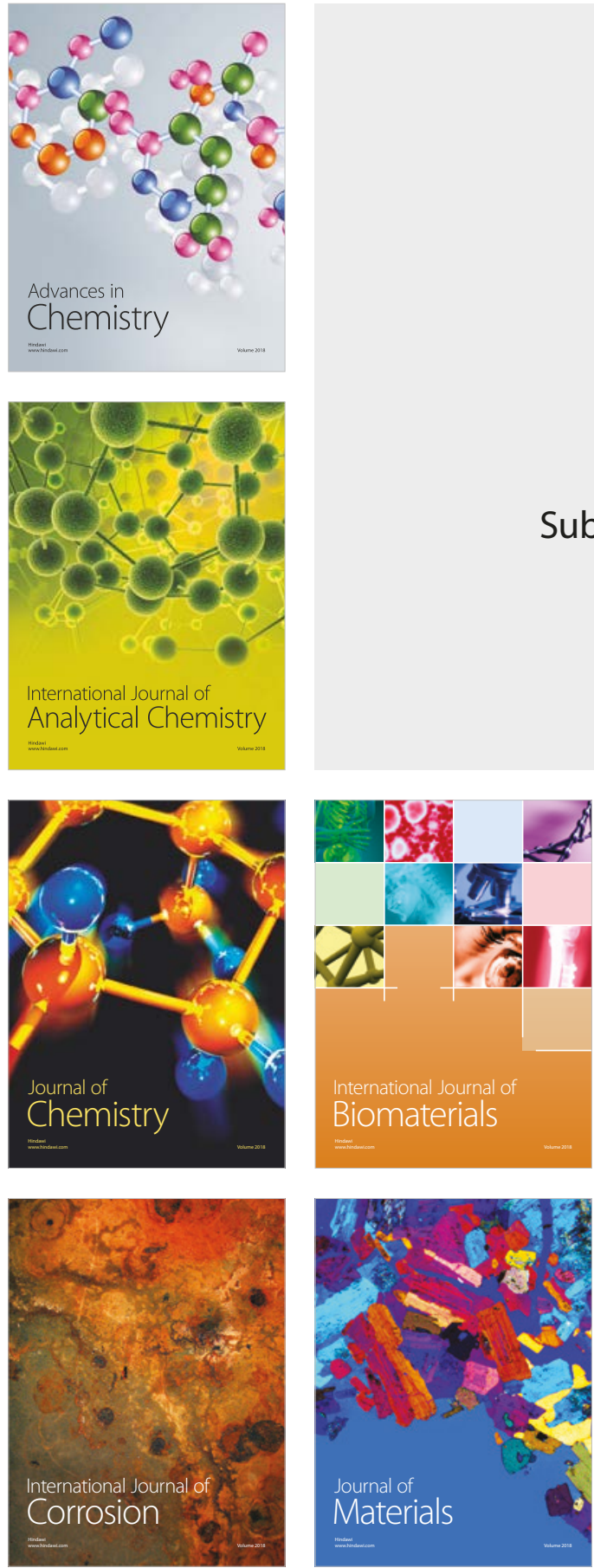

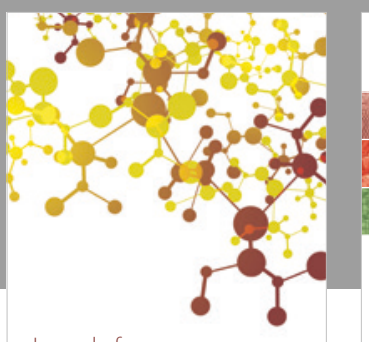

Journal of

Applied Chemistry
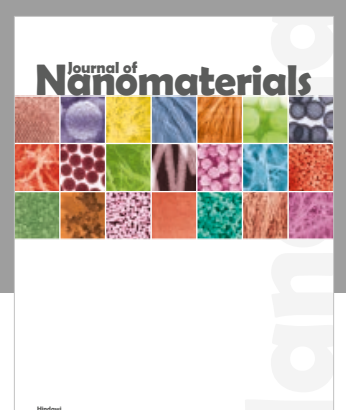

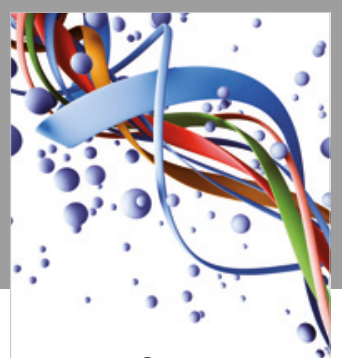

Scientifica

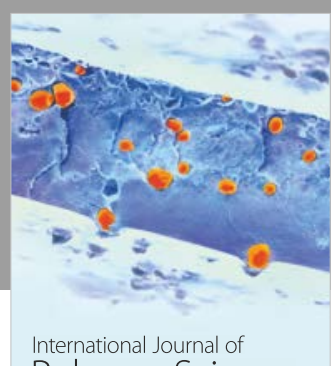

Polymer Science

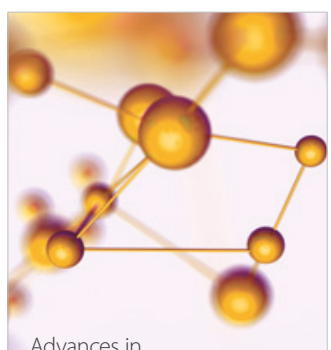

Physical Chemistry
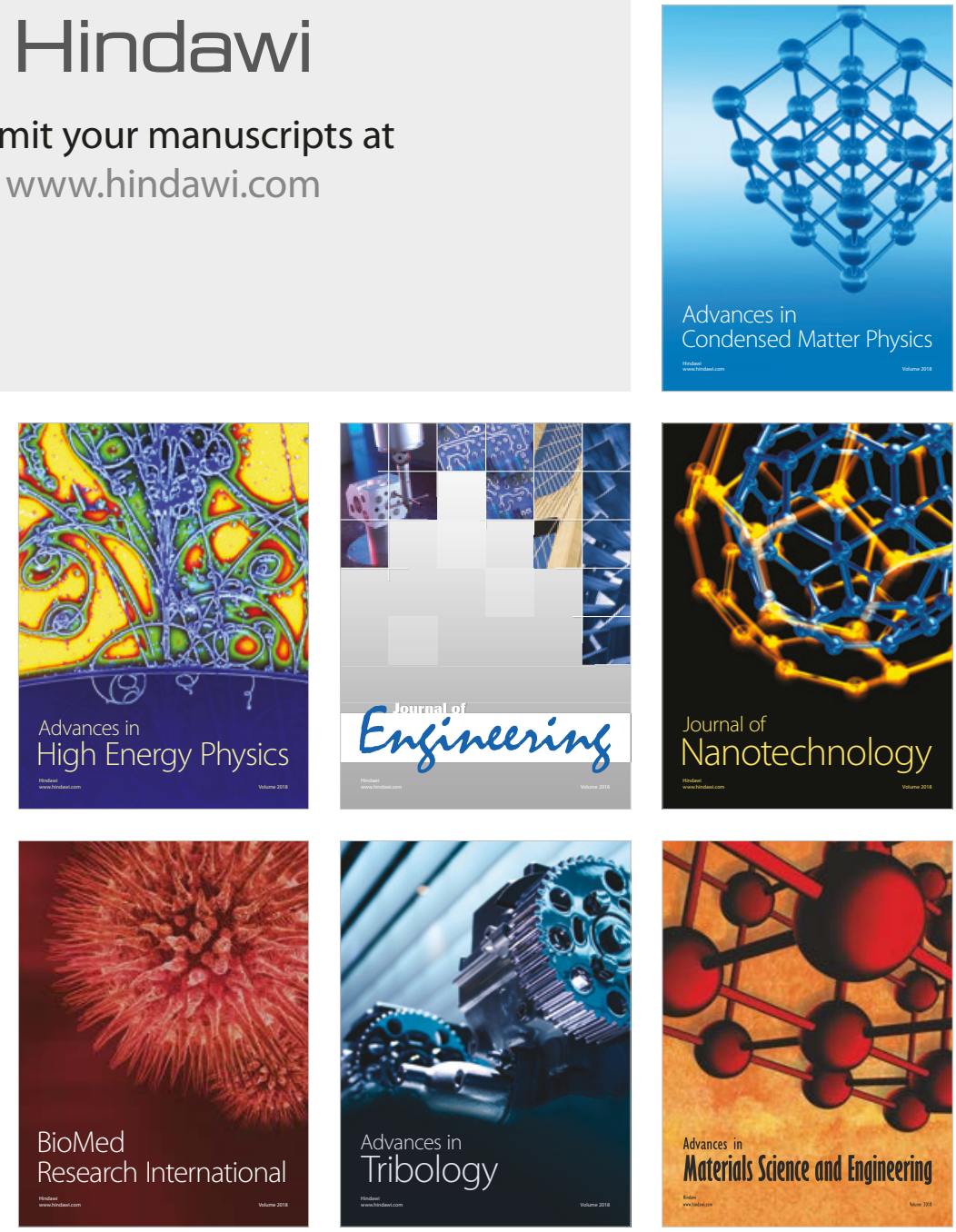